\title{
ANÁLISIS DE LA IMPLEMENTACIÓN INSTITUCIONAL DE LA MODALIDAD B-LEARNING EN CARRERAS DE PREGRADO DE TRES UNIVERSIDADES CHILENAS $^{1}$
}

\author{
Paola Costa ${ }^{2}$, \\ Karem Celis ${ }^{3}$, \\ Nancy Castillo-Valenzuela ${ }^{4}$, \\ Gabriel Espinoza ${ }^{5}$
}

\section{RESUMEN}

¿Cómo las instituciones de educación superior están implementando la modalidad blendedlearning o b-learning en pregrado? Es la pregunta que orienta este estudio y que busca ser respondida a través del análisis de contenido de nueve entrevistas a responsables institucionales de tres universidades chilenas. Para el efecto de este estudio, el concepto de "dispositivo de formación híbrido" (el foco del proyecto europeo de investigación Hy-Sup) (Deschryver \& Charlier, 2012) en lugar de b-learning, resultó pertinente para abordar la pregunta desde una perspectiva antropocentrista, anclada en la institución, docentes y estudiantes y no en el objeto técnico en sí. A su vez, este marco conceptual favoreció la realización de análisis comparativos de los resultados con los obtenidos en la investigación Hy-Sup, en el contexto europeo. Los resultados muestran que las diferencias entre la visión y acciones implementadas en las instituciones podrían condicionar los tipos de dispositivos de formación híbridos desarrollados. La investigación busca aportar a estudios futuros con una caracterización y conceptualización de estas formaciones, a fin de posibilitar el análisis de su impacto en los aprendizajes de los estudiantes, así como en las prácticas pedagógicas de los docentes.

Conceptos clave: b-learning, blended learning, educación superior, pregrado, semipresencial, tecnología.

\section{ANALISIS OF THE IMPLEMENTATION OF THE B-LEARNING MODALITY IN UNDERGRADUATE PROGRAMS AT THREE CHILEAN UNIVERSITIES}

\section{ABSTRACT}

How are higher educational institutions implementing the blended learning, b-learning modality in their undergraduate degree programs? This inquiry guides the present study and looks to be answered through a content analysis of nine interviews applied to those who oversee this process in three Chilean Universities.

\footnotetext{
Proyecto apoyado financieramente por CNED/ Convocatoria 2017.

Universidad Santo Tomás, Santiago, Chile. Contacto: pcostac@santotomas.cl

Universidad Santo Tomás, Santiago, Chile. Contacto: karemcelis@santotomas.cl

Universidad del Bío Bío, Chillán, Chile. Contacto: nacastil@ubiobio.cl

Universidad Santo Tomás, Santiago, Chile. Contacto: gespinozal2@santotomas.cl
} 
For the purpose of this study, the concept of "hybrid systems" (the focus of the European research project, Hy-Sup) (Deschryver \& Charlier, 2012) is used instead of b-learning, in order to answer the study's main objective from an anthropocentrist perspective, which focuses on the institution, professors and students, rather than on the technical object. In turn, this conceptual framework allows for the comparison of the results of this study with those obtained by the Hy-Sup research, in the European context. The study's results show that differences between the institutional vision and the actions taken might determine the type of hybrid systems developed. This research aims to contribute to future studies by characterizing and conceptualizing these types of "systems", allowing for an analysis of their impact on student learning, as well as on professors' pedagogical practices.

Key concepts: b-learning, blended learning, higher education, hybrid systems, undergraduate degree, technology.

\section{Introducción}

Este artículo presenta los resultados de la fase cualitativa de una investigación que utilizó técnicas mixtas y cuyo objetivo fue analizar los procesos institucionales de implementación de los dispositivos de formación híbridos en carreras de pregrado de tres universidades chilenas. En la etapa cuantitativa fueron encuestados 130 docentes y 391 estudiantes de siete carreras de pregrado (Pedagogía General Básica, Pedagogía en Inglés, Educación Parvularia, Bachillerato en Ciencias, Trabajo Social, Psicología, Ingeniería Civil Industrial). Los resultados permitieron validar la herramienta de recolección de datos de la investigación europea Hy-Sup (Deschryver \& Charlier, 2012), así como caracterizar las formaciones soportadas por un entorno virtual de aprendizaje.

La fase cualitativa — que se presenta en este artículo- tuvo como objetivo identificar las estrategias institucionales implementadas por tres universidades y evaluar cómo estas favorecen o impiden el desarrollo de la modalidad b-learning en carreras de pregrado. Para ello, fueron entrevistados un total de nueve responsables institucionales de la implementación de la modalidad b-learning pertenecientes a tres universidades.

A través de una revisión de la literatura, el estudio muestra la importancia de contar con una definición epistemológica de los conceptos asociados a b-learning y a las plataformas virtuales de 
aprendizaje, ya que su indefinición puede repercutir en el estudio de sus efectos. Esta consideración también es válida para el análisis de las estrategias y acciones institucionales, declaradas por los responsables en las universidades, referidas al desarrollo de dispositivos de formación híbridos, las que fueron el objeto de estudio de la presente investigación. Cabe destacar que la estrategia y las acciones que cada institución adopte en relación con la integración de aulas virtuales en las clases presenciales podría jugar un rol determinante en el éxito o fracaso de la modalidad b-learning en pregrado (Mazohl \& Makl, 2016; Porter, Graham, Bodily \& Sandberg, 2016; Van der Linden, 2014). En este sentido, realizar una caracterización de la existencia de una estrategia institucional en relación con estas formaciones es útil para contextualizar los tipos de dispositivos de formación híbridos, así como para analizar su implementación y sus efectos.

\section{Interés por el uso y desarrollo de los dispositivos de formación híbridos}

En los últimos años los investigadores en tecnología educativa se han interesado por analizar los posibles cambios que podría introducir el uso de plataformas virtuales de aprendizajes en la educación superior (Area, San Nicolás y Sanabria, 2018; Deschryver \& Charlier, 2012; Peraya \& Campion, 2007). Una reciente revisión de la literatura (Marín, Duart, Galvis \& Zawacki-Richter, 2018) y el análisis cualitativo de los artículos científicos publicados entre 2004 y 2017 en el International Journal of Educational Technology in Higher Education (ETHE) muestra que en el período 2016-2017 la mayoría de las investigaciones en tecnología educativa en educación superior "han centrado su interés en el estudio del diseño y desarrollo de estrategias y actividades con uso de entornos y herramientas digitales" (Marín et al., 2018, p. 5). Los autores del análisis destacan que los términos "desarrollo" y "uso" adquieren mayor relevancia en estos últimos años en relación con los años anteriores analizados (2004-2015).

En Chile, una revisión de la literatura atisba que las investigaciones realizadas sobre b-learning en los últimos 10 años no entregan una visión general del desarrollo de esta modalidad en 
pregrado y se concentran en estudios de caso que entregan resultados aislados y no generalizables a otros contextos (Garrido, 2009; Mendiburo-Seguel y Reininger, 2011; Navarrete, Aburto y Fuentes, 2016).

\section{Hacia una definición que permita caracterizar los dispositivos de formación híbridos}

La mediatización y la puesta a distancia de los cursos presenciales se ven facilitados por el uso de plataformas virtuales de aprendizaje. Así, es posible analizar estas formaciones desde la perspectiva de la modalidad semipresencial, blended-learning o b-learning. Sin embargo, la revisión de la literatura muestra que no existe un consenso en la definición del término (Charlier, Deschryver \& Peraya, 2006). Las definiciones más citadas integran elementos como: el uso de la tecnología, la distribución del tiempo virtual-presencial y la combinación de diferentes métodos de enseñanza. Así, por ejemplo, Bartolomé (2008) define el b-learning como "un sistema en el que se mezclan situaciones de presencialidad y no presencialidad, recurriendo a las tecnologías más adecuadas para cada necesidad" (p. 15). Para Glazer (2011) el b-learning consiste en una mezcla de los dos medios (face to face y online), donde ambos se refuerzan mutuamente y crean un solo curso unificado. Sin embargo, ninguna de estas definiciones da cuenta de todas las características específicas de una formación en modalidad b-learning.

Charlier et al. (2006) constataron que la falta de conceptualización y de definición de las características del b-learning repercutía en el análisis de los efectos de este tipo de formaciones. Por ello, en 2006 comenzaron un trabajo de redefinición del concepto. Estos autores decidieron adoptar el término híbrido (en referencia al origen biológico del término), en reemplazo del b-learning, con el fin de poner en relieve que el porcentaje de presencia y distancia no es lo que más caracteriza este tipo de formaciones, sino que se trata de una modalidad nueva, susceptible de producir nuevos efectos en los aprendizajes. En consecuencia, con esta visión Charlier et al. (2006) definen los dispositivos de formación híbridos en función de las dimensiones que los caracterizan: 
Un dispositivo híbrido se caracteriza por la presencia en un dispositivo de formación de dimensiones innovadoras, asociadas a la distancia. El dispositivo es híbrido, porque supone la utilización de un entorno tecno-pedagógico y se sostiene en formas complejas de mediación y mediatización (p. 481).

A nivel teórico, la noción de dispositivo de formación tiene asidero en los conceptos de "dispositivo de formación" y de "dispositivos de comunicación y de formación mediatizadas", basadas en la evolución de la definición de "dispositivo" elaborada por Michelle Foucault en los años setenta. Analizando la genealogía del término - y el vínculo con Aristóteles, Hegel y Heidegger-, Agamben (2011) señala que, si bien la complejidad del concepto tiende a abordar diversas dimensiones de fenómenos vinculados con el poder, este se puede sintetizar como: "todo aquello que tiene, de una manera u otra, la capacidad de capturar, orientar, determinar, interceptar, modelar, controlar y asegurar los gestos, las conductas, las opiniones y los discursos de los seres vivos" (Agamben, 2011, p. 257). De esta manera, la noción de dispositivo está caracterizada por dinámicas vinculadas con la articulación de acciones y elementos técnicos que conducirán o disciplinarán a distintos agentes para la prosecución de objetivos determinados. En los años ochenta, los investigadores en ciencias de la educación francófonos adoptaron el concepto de "dispositivo de formación", entendiéndolo como: "los lugares, los métodos y el conjunto de actores y medios movilizados para alcanzar un objetivo" (Albero, 2010, p. 47). Bajo esta definición adoptar el término de dispositivo de formación, permite situar el análisis desde una perspectiva que toma en cuenta variables individuales y contextuales y no las restringe al estudio del artefacto tecnológico por sí mismo ni a la sola interacción de los estudiantes y docentes con el artefacto.

La traducción del término "dispositivo de formación híbrido" al inglés, no ha estado exenta de discusiones teórico-metodológicas. Para la investigadora suiza, Bernadette Charlier (2019), el concepto que más se aproxima es el de learning environmment o en español ambiente de aprendizaje, sin embargo, aclara que solo es homologable en el sentido de posibilitar el análisis de los efectos desde un modelo 
de causalidad circular, sin dejar fuera del análisis toda la complejidad de la problemática (los individuos, recursos humanos materiales y humanos puestos a disposición, la interacción de los elementos, las estrategias y los aprendizajes, entre otros), así como los diferentes niveles (micro, meso, macro) y variables que intervienen en el proceso. En definitiva,

se trata de recoger un conjunto de datos que caractericen a los individuos, sus interacciones con los entornos virtuales de aprendizaje, sus estrategias y los productos del aprendizaje, tal cual como son reconocidos por ellos u objetivados por la institución (Charlier, 2019, p. 98).

Cabe destacar que los autores de la definición de dispositivo de formación híbrido participaron del proyecto de investigación Hy-Sup (Deschryver \& Charlier, 2012) en el que colaboraron seis universidades europeas con la finalidad de caracterizar los dispositivos híbridos, así como analizar sus efectos. En este estudio, la definición creada en 2006 evolucionó, eliminando la dimensión de innovación asociada a la distancia, ya que el uso de las plataformas virtuales se ha generalizado tanto en los últimos años que ya no son ninguna novedad. Asimismo, agregan que los dispositivos híbridos son diferentes entre sí, porque reflejan las elecciones de sus creadores, las que estarían a su vez relacionadas con: "las percepciones sobre la enseñanza y el aprendizaje, el control y la apertura del dispositivo de formación, la organización del espacio y del tiempo, así como del rol de los medios en los procesos de aprendizaje" (Deschryver \& Charlier, 2012, p. 85). Esta definición ha permitido elaborar una matriz de análisis de los dispositivos de formación híbridos, favoreciendo la proliferación de estudios comparados, así como el desarrollo de tipologías de uso y de análisis de los efectos (Burton et al., 2011; Deschryver, 2008; Peraya \& Peltier, 2012).

\section{Perspectivas de análisis de los dispositivos de formación híbridos}

En un análisis más específico de la literatura en tecnología educativa, y con el fin de relevar el hallazgo y enfoque del presente estudio, 
se resumen a continuación los abordajes predominantes en las investigaciones de los llamados dispositivos de formación híbridos.

a. Las variables que influyen en la aceptación de la modalidad b-learning

Estas han sido estudiadas por numerosos autores (Long, Cummins \& Waugh, 2016; Martín García, García del Dujo y Muñoz, 2014; Nguyet, Zhu, Struyven \& Blieck, 2016; Porter et al., 2016; RiveraLaylle, Fernández, Guzmán y Pulido, 2017), así como también lo han sido los análisis de casos exitosos de implementación de esta modalidad en la enseñanza superior (Cher \& Wang, 2016; Martín García et al., 2014; Mejía et al., 2017; Turpo, 2012). Entre las variables individuales que influenciarían en el rechazo o aceptación de un modelo de formación en modalidad b-learning, evidenciadas por estos estudios están:

- las desigualdades en las competencias digitales, tanto de estudiantes como de los docentes;

- el acceso desigual a las tecnologías;

- el sentimiento de autoeficacia personal para el uso de las tecnologías;

- las creencias respecto de la utilidad y la eficacia para la mejora de los aprendizajes de una formación en modalidad b-learning y "expectativa de resultados" (Martín García et al., 2014; Nguyet et al., 2016; Rivera-Laylle et al., 2017).

\section{b. Estrategias didácticas y modelos de aprendizaje}

Estos estudios analizan principalmente métodos que se podrían ver facilitados por el uso de plataformas virtuales como, por ejemplo, el aula invertida ${ }^{6}$. Destacan los trabajos de Lebrun, Gilson y Goffinet (2017), quienes elaboraron una tipología de clases invertidas,

6 "Si bien existen múltiples formas de practicar la clase invertida, todas se apoyan en grados diversos en estos principios fundamentales: aumentar las interacciones entre docentes y estudiantes, así como entre los estudiantes, individualizar los aprendizajes, desarrollar los métodos activos y una pedagogía colaborativa. En definitiva, crear un ambiente de aprendizajes y de actividades donde los estudiantes desarrollen la autonomía y la responsabilidad de sus propios aprendizajes" (Peraya, 2015 p.8). 
distinguiendo aquellas centradas en la enseñanza y aquellas que están focalizadas en el estudiante (aprendizaje). Los tipos de clases invertidas identificadas por estos autores abarcan desde la concepción más clásica "Lectures at home and homework in class" a una versión enriquecida.

Otros autores analizan las variables individuales y contextuales que influyen en los resultados que se obtienen en los aprendizajes de los estudiantes, utilizando la estrategia de aula invertida (Schwarzenberg, Navon, Nussbaum, Pérez-Sanagustín \& Caballero, 2017), otorgándole una fuerte importancia a la articulación de la presencia y la distancia, y a la retroalimentación. Por su parte, Yi (2018)" pone en evidencia los principales problemas que encuentran los académicos de una universidad del norte de China, para implementar el aula invertida en sus clases. Sus resultados coinciden con los de otros autores (Long et al., 2016) y muestran que invertir el aula requiere de un esfuerzo mayor de planificación tecno-pedagógica por parte de los docentes y de una participación activa de los estudiantes, quienes muchas veces no están dispuestos a abandonar modelos de enseñanza aprendizaje tradicionales (Karsenti, Larose y Núñez, 2002; Lamine \& Abdid, 2016).

En los últimos años, investigadores han comenzado a integrar técnicas de analítica de aprendizajes (learning analytics), para analizar el impacto de la clase invertida en los procesos de enseñanza aprendizaje, proponiendo la elaboración de herramientas que faciliten a los docentes la visualización de estos datos para tomar decisiones pedagógicas que favorezcan la adaptación de las actividades al ritmo y necesidades de los estudiantes (Jovanovic, Gasevic, Dawson \& Mirriahi, 2017; Lucke, 2014; Pierrot, El-Kechai, Iksal \& Cerisier, 2017).

En cuanto al análisis del trabajo colaborativo en b-learning, destacan los trabajos de Deschryver (2008) y Quintin (2008), quienes muestran que las interacciones sociales y la colaboración en los dispositivos de formación híbridos tendrían una incidencia en el desarrollo de un enfoque de aprendizaje en profundidad, así como en la disminución del sentimiento de aislamiento, propio de las formaciones a distancia. 
c. Impacto de los dispositivos de formación híbridos en los procesos de enseñanza aprendizaje

La gran mayoría de los estudios centra su interés en evaluar el rendimiento académico de los estudiantes y en la comparación de estos resultados con un grupo de control (Delgado-Almonte, Andreu \& Pedraja-Rejas, 2010; Means, Toyama, Murphy \& Baki, 2013; Mendiburo-Seguel \& Reininger, 2011; Vásquez-González, Pleguezuelos y Cabas, 2011). Sin embargo, no logran resultados concluyentes que demuestren que la modalidad b-learning en educación esté correlacionada estadísticamente con mejores resultados académicos.

Otros estudios intentan evaluar el impacto del b-learning a través de las percepciones en torno a la satisfacción de los estudiantes y/o de los docentes (Sanabria, Castro, Padrón y Area, 2013; Vásquez, 2014) evidenciado la resistencia de los estudiantes y de los docentes a modificar sus enfoques de aprendizaje, siendo la formación presencial más valorada en pregrado que aquellas que integran un grado de distancia. En algunas carreras, el b-learning es valorado por la flexibilidad que permite (movilidad y asistencia) y por el atractivo de utilizar tecnologías digitales para el aprendizaje (motivación y desarrollo de habilidades digitales).

Es posible encontrar investigaciones que identifican efectos potenciales en los aprendizajes percibidos por los participantes de una formación en modalidad b-learning, ya sea en sus dinámicas identitarias, en las interacciones sociales y en el surgimiento de comunidades de prácticas, así como en la apropiación por parte de los docentes de dispositivos tecno-pedagógicos y sus efectos en las prácticas pedagógicas (Peraya, Charlier \& Deschryver, 2014). Sin embargo, sus resultados no son generalizables, ya que corresponden a estudios acotados a contextos particulares y con una población limitada.

La caracterización de los dispositivos de formación híbridos, realizada por la investigación Hy-Sup (Deschryver \& Charlier, 2012), permitió identificar cinco dimensiones al respecto: 
- articulación de la presencia-distancia;

- acompañamiento humano;

- uso del entorno digital tecno-pedagógico;

- mediatización y mediación instrumental; y

- grado de apertura del dispositivo.

Los resultados de esta investigación muestran que la articulación de la presencia y de la distancia, característica de los dispositivos de formación híbridos, estará fuertemente asociada con el modelo pedagógico predominante en las representaciones de los docentes. Por ejemplo, si el modelo predominante es de tipo constructivista los docentes les asignarán mayor importancia a las actividades realizadas fuera de la sala de clases que favorezcan en sus estudiantes el tratamiento de la información, el desarrollo de la autonomía y la colaboración entre pares. Por el contrario, si los docentes privilegian un modelo tradicional o transmisivo, valorarán más las clases presenciales y motivarán a sus estudiantes a realizar actividades de "toma de apuntes, memorización, entrega de informaciones" (Deschryver, 2008). Los resultados del proyecto de investigación europeo Hy-Sup (Deschryver \& Charlier, 2012) permitieron identificar seis tipos de dispositivos híbridos, tres de ellos centrados en la enseñanza y tres en el aprendizaje. Esta tipología ha facilitado el análisis del impacto en los aprendizajes de los estudiantes de los tipos de dispositivo de formación híbrido con los tipos de aprendizaje (superficial o en profundidad) de los estudiantes (Deschryver \& Charlier, 2012; Lebrun et al., 2017).

\section{d. Apoyo institucional}

Existe consenso en la literatura acerca de la necesidad de contar con una estrategia y con apoyo institucional para dar las facilidades (de tiempo y recursos) y el reconocimiento necesario a los docentes que se formen en modalidad b-learning, así como aquellos que implementen esta modalidad con sus estudiantes (Albero, 2014; Albero, Linard \& Robin, 2009; Porter et al., 2016; Van der Linden, 2014). 


\section{Operacionalización y pertinencia del concepto de dispositivos de formación híbridos}

El presente estudio no busca caracterizar los modelos de aceptación de las tecnologías por parte de los usuarios (Davis, 1985; Lai, 2017), ni tampoco las características de los artefactos utilizados, sino explorar cómo se construye la implementación de los dispositivos híbridos por parte de las universidades en pregrado, considerando la complejidad de las variables involucradas en el proceso, desde sus niveles micro (los actores), meso (la institución) y macro (la sociedad). Esto último, sostiene la hipótesis misma del concepto de dispositivo de formación híbrido, y la pertinencia de su uso, que sobrepasa el análisis del artefacto, para centrarse en la actividad humana (Léontiev, 1975; Rabardel, 1995; Vygotski, 1985). Además, la definición de este concepto es sustentada por los autores del proyecto Hy-Sup (Deschryver \& Charlier, 2012), lo que permitirá conocer los procesos institucionales de implementación de los dispositivos híbridos en carreras de pregrado de universidades chilenas y comparar los resultados obtenidos en el contexto nacional respecto del europeo.

Resulta necesario recordar que la adopción de nuevas estrategias de enseñanza-aprendizaje, así como la integración de las tecnologías en el aula, no obedece únicamente a la voluntad de los docentes. El análisis de la literatura le asigna una gran relevancia a variables contextuales que favorecerían la adopción de nuevas metodologías de enseñanza, entre las que destaca fuertemente el contexto institucional (Mazohl \& Makl, 2016; Porter et al., 2016; Van der Linden, 2014).

\section{Metodología}

El presente estudio corresponde a los resultados cualitativos de una investigación de mayor alcance, realizada en tres universidades chilenas. Tanto la información cualitativa, como la cuantitativa fue recopilada mediante los instrumentos de recolección de datos aplicados por el proyecto de investigación europeo Hy-Sup (Deschryver \& Charlier, 2012), los que fueron adaptados para su validación en el contexto nacional. 
La sección cualitativa del estudio se focaliza en el análisis de contenido de entrevistas realizadas a los responsables institucionales de tres universidades acerca de la implementación de la modalidad b-learning en pregrado. La estrategia metodológica de los resultados expuestos en este artículo se concentra en un enfoque de tipo cualitativo, que busca conocer el sentido de las acciones y los procesos ejecutados por los actores frente a un fenómeno en particular: en este caso, la implementación de los dispositivos híbridos en tres universidades chilenas. Los hallazgos del estudio ejecutado por el Hy-Sup, por Douzet, Letor y Ronchi (2012) servirán para realizar el contraste y la discusión. La selección se justifica debido a que la herramienta de recolección de datos corresponde a la de dicho estudio, lo que favorece la homologación y el contraste entre contextos y resultados.

La investigación entiende su condición exploratoria y no generalizable, pero encuentra su pertinencia en la complejización del abordaje de estos procesos en instituciones de educación superior en Chile, además de permitir un marco comparativo con instituciones de educación superior europeas.

\section{Muestra y recolección de datos}

El muestreo llevado a cabo fue de tipo intencionado, entendiendo una justificación de tipo teórica en la cual los investigadores pueden realizar una primera discriminación que defina la muestra, pero no determinar los resultados de la información a obtener (Coyne, 1997). En este sentido, su pertinencia es establecer lineamientos comunes para realizar el estudio y, desde ahí, enriquecer el fenómeno desde las posibles emergencias de la información recopilada (Berg, 2009). El muestreo intencionado, en este caso, resulta pertinente debido a las categorías entendidas como relevantes para estudiar el fenómeno, las que se basan en los criterios teóricos del enfoque dominante de esta investigación. Los criterios de selección de los participantes fueron:

- Universidades chilenas que contaran con entornos virtuales de aprendizaje como apoyo a la docencia de pregrado.

- Universidades que contaran con diversas áreas de formación y presencia en diferentes regiones. 

EN CARRERAS DE PREGRADO DE TRES UNIVERSIDADES CHILENAS - P. Costa, K. Celis, N. Castillo-Valenzuela, G. Espinoza

- Disposición de las universidades para trabajar con los investigadores durante el período de investigación.

Frente a los criterios establecidos — homologados a los del proyecto Hy-Sup - fueron entrevistados los directores de las unidades relacionadas con la virtualización de las formaciones y asesores técnico-pedagógicos de las tres instituciones. En la selección de la muestra, no fueron relevantes otras características individuales de los sujetos entrevistados como, por ejemplo: género, nacionalidad, edad, formación, etc. Fueron elegidas tres universidades del país, procedentes de distintas regiones (de Valparaíso, del Biobío y de la Región Metropolitana) pertenecientes y no pertenecientes al CRUCH, con presencia regional y/o nacional, que además presentaran diferencias sustantivas en sus matrículas totales ${ }^{7}$. Una de las universidades opera en 12 regiones. Las otras dos se concentran en: una región de la zona centro, mientras que la otra lo hace en la zona centro-sur del país.

La fase de recolección de datos fue realizada el segundo semestre del año académico 2018 (julio-septiembre), a través de entrevistas semiestructuradas, las que fueron aplicadas a nueve responsables institucionales del desarrollo e implementación (tres de cada universidad) de lo que — bajo la propuesta teórica del presente estudio— se entenderá como dispositivo de formación híbrido.

El modelo de la entrevista se basó en el trabajo de Douzet et al. (2012) descrito en el informe final del proyecto Hy-Sup (Deschryver $\&$ Charlier, 2012). En consecuencia, las entrevistas fueron guiadas en función de las categorías:

- Visión institucional.

- Cultura tecnológica.

- Gestión de la calidad y evaluación de la enseñanza-aprendizaje. 
Paralelamente, se analizó cómo estas repercutían en las variables intermedias:

- Apoyo financiero.

- Apoyo técnico-pedagógico.

- Reconocimiento institucional.

El objetivo fue evaluar finalmente los impactos percibidos de los dispositivos híbridos en la movilidad e intercambio interinstitucional y los usos observados.

En el análisis de los resultados se exploró, además, si las variables diferenciadoras de las universidades como: localización geográfica, diferencias en las matrículas, tipo de dependencia (estatal, privada, con o sin aporte del Estado) tenían alguna incidencia en la estrategia institucional adoptada y si el dispositivo de formación híbrido constituía o no (según la percepción de los entrevistados) una práctica arraigada en la institución. Para ello, se siguió el modelo teórico propuesto por diversos autores (Bonamy, Charlier \& Saunders, 2002; Deschryver, 2008; Peraya, Charlier \& Deschryver, 2006) en el que se distinguen tres tipos de prácticas, según la posición del dispositivo de formación híbrido en relación con las prácticas dominantes de la institución (Costa, Peraya y Rizza, 2016):

- de enclave: las prácticas analizadas son disruptivas y no generalizadas, por lo que coexisten con las prácticas dominantes;

- tete de point: se favorece el desarrollo de nuevas prácticas que afectan a las que dominan en la institución; 
- arraigada: las prácticas antes nuevas, se han generalizado y son las que dominan en la institución.

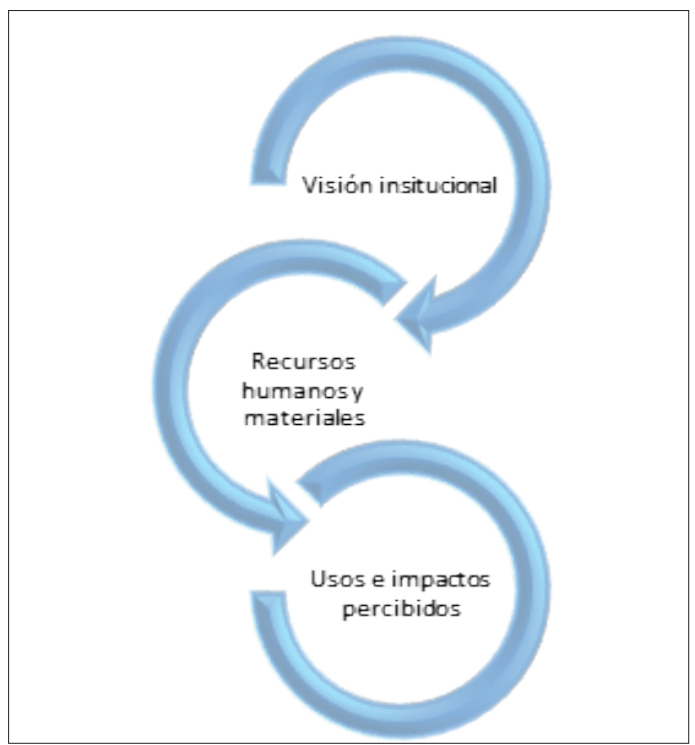

Figura 1. Esquema de análisis de categorías.

Fuente: Elaboración propia.

Si bien el enfoque de Douzet et al. (2012) apunta a un análisis "institucional" en un sentido teórico, el presente entiende la acción institucional desde los objetivos y controversias asociadas al desarrollo de los dispositivos de formación híbridos. Este abordaje se justifica a través de la noción de controversia que plantea Latour (1988) y el desarrollo de su pertinencia en procesos institucionales (Swan \& Scarbrough, 2001; Tsoukas \& Vladimirou, 2001). Lo anterior permite concentrar el estudio en los procesos productivos de conocimiento, decisiones, definiciones y los valores que los diversos actores involucrados van adoptando frente al mismo fenómeno.

Con el objetivo de analizar la estrategia institucional y el contexto de desarrollo de los dispositivos de formación híbridos en las tres universidades, se realizaron las siguientes acciones:

- Se preparó una entrevista semiestructurada siguiendo las categorías antes descritas, a fin de responder al objeto de esta 
fase. Para ello, se siguió la pauta utilizada en el proyecto Hy-Sup.

- Tres investigadores chilenos, no solo expertos en la temática, sino también en metodología cualitativa validaron las preguntas de la guía de entrevista. Se realizaron mejoras en la redacción y claridad de las mismas.

- Se llevaron a cabo entrevistas individuales a tres actores clave en la implementación del b-learning por universidad (nueve en total). Los entrevistados fueron directores y asesores técnicopedagógicos de las tres universidades.

\section{Análisis de datos}

El análisis de las entrevistas consideró diferentes etapas, y fue apoyado por el software ATLAS.ti versión 8. Esta técnica resulta pertinente por su ductilidad para indagar en los contenidos, los elementos simbólicos de lo enunciado y la intencionalidad de las producciones discursivas de los actores (Arfuch, 2002; Sayago, 2014). Criterios que resultan relevantes para entender cómo se produce, sustenta y reflexionan las estrategias institucionales.

Las etapas consideradas en el análisis fueron las siguientes:

- Lectura global de las entrevistas, en conjunto con tres investigadores;

- codificación de citas con el uso de software ATLAS.ti versión 8;

- categorización de las citas, considerando categorías predeterminadas por el marco teórico, así como por la presencia de categorías emergentes;

- finalmente, el análisis e interpretación de resultados.

\section{Aspectos éticos}

Durante el proceso de investigación, se fueron tomando medidas de control de confiabilidad y validez, tanto en la construcción de los instrumentos de recolección de datos utilizados en las distintas etapas, como en la verificación de los resultados obtenidos a través de los distintos métodos y de la triangulación de sus resultados. Por 
ejemplo, todas las entrevistas fueron realizadas por un investigador externo a las tres casas de estudio. De igual manera, un asistente de investigación realizó las transcripciones totalmente anónimas. Se tuvieron presentes consideraciones de tipo ético (entre ellas, solicitud de consentimiento informado a los entrevistados, confidencialidad de la información, etc.), con el fin de proteger la integridad de los participantes y mantener estándares altos de rigor investigativo. Con el fin de garantizar el anonimato de los entrevistados estos fueron codificados con números y las universidades con letras A, B y C. De esta forma, los entrevistados de la Universidad A, corresponden a los entrevistados 1, 3 y 5; los de la Universidad B a entrevistados 2, 4 y 6 y los de la Universidad C a los entrevistados 7, 8 y 9.

\section{Resultados}

Los resultados se presentan de acuerdo con las categorías preestablecidas en la guía de entrevista, a saber: visión institucional, cultura tecnológica y gestión de la calidad y evaluación de la enseñanza-aprendizaje.

También se analizó cómo estas categorías repercutían en el apoyo financiero y técnico-pedagógico y en el reconocimiento institucional, de modo de evaluar finalmente los impactos percibidos en los dispositivos híbridos en la movilidad e intercambio interinstitucional y los usos observados del aula virtual.

Esta última categoría fue emergente en el contexto del estudio y surgió espontáneamente en el discurso de los nueve responsables institucionales entrevistados.

En el análisis de resultados no se encontraron diferencias que tuvieran relación con características específicas de la muestra como son: localización geográfica, tipo de dependencia o número de estudiantes matriculados. Las diferencias existentes están más bien en la visión estratégica y en los recursos tanto materiales como humanos asignados por cada institución para lograr los objetivos propuestos. Cabe destacar que en las Universidades A y B, los dispositivos de formación híbridos están situados, según 
la percepción de los entrevistados, en tete de point, es decir, que la institución impulsa el desarrollo de este tipo de prácticas, pero no son aún las dominantes. Sin embargo, estarían afectando a las prácticas antiguas. Así, por ejemplo, los docentes que no usan el aula virtual son presionados a hacerlo por sus estudiantes, colegas y/o superiores jerárquicos. En tanto, la Universidad C es la única de las tres en las que los dispositivos de formación híbridos constituyen una práctica "arraigada" en la institución y es la única que cuenta con una dirección específica para el desarrollo de este tipo de programas en pregrado, dependiente directamente de la Vicerrectoría Académica y lleva funcionando 20 años.

Cabe desatacar que las tres instituciones se encuentran desarrollando una oferta formativa para estudiantes de pregrado, con asignaturas $100 \%$ online y b-learning, a partir del año académico 2019. Si bien en la Universidad C esta oferta ya existe, se han planteado el desafío de aumentarla considerablemente en los próximos años:

tenemos que de aquí al próximo plan estratégico en nuestro proceso de acreditación institucional mostrar que al menos el 20\% de nuestras asignaturas son semipresenciales (Participante 1, Universidad C).

\section{a. Visión institucional: estrategias y acciones}

La visión acerca del desarrollo de dispositivos de formación híbrida o formaciones en modalidad b-learning en pregrado no está declarada explícitamente en dos de las tres instituciones:

falta poder plasmar estas acciones en un documento (Participante 6, Universidad B).

hoy en día no es parte del plan estratégico (Participante 1, Universidad A).

Esta falta de definición institucional se ve reflejada también en los términos utilizados por los entrevistados para referirse a los dispositivos de formación híbridos: b-learning, semipresencial, cursos 
online, flex class, formaciones híbridas, cursos presenciales con apoyo virtual, fueron algunos de los conceptos utilizados indistintamente por estos.

Contar con una visión institucional explícita respecto de los dispositivos de formación híbridos es una necesidad expresada en las dos instituciones que no la poseen. En ambas universidades el tema es discutido por las autoridades y se encuentran trabajando en ello:

A nivel de Rectoría y de Vicerrectoría es una discusión que está muy presente y está presente en todas las discusiones que involucran la planificación estratégica (Participante 1, Universidad A).

el rector, vicerrector y el Consejo Académico Superior, ya estamos muy convencidos que este es el camino, queremos declararlo y por eso todos los proyectos de capacitación, de servicios, de avanzar en talleres virtuales 100\% y queremos hacerlo bien, así que es algo que estamos trabajando fuerte en el proceso de desarrollo 2019-2023 (Participante 3, Universidad A).

En la Universidad B, la discusión también se da a nivel de la Vicerrectoría Académica, aunque aún no se ha llegado a plasmar en el plan estratégico:

a nivel de Vicerrectoría se discute, pero formalmente no se ha llegado a un documento (...) pero sí están las experiencias e iniciativas (Participante 6, Universidad B).

La Universidad C es la única de las tres instituciones que ha avanzado en la elaboración de documentos institucionales que explicitan y difunden tanto la visión institucional como las acciones a seguir para desarrollar dispositivos de formación híbridos. Para ello destinan los recursos humanos y materiales necesarios y se perciben a sí mismos como referentes en la materia en Chile, dada su experiencia de 12 años impulsando la innovación pedagógica, a través de la integración de las tecnologías digitales como apoyo a la docencia universitaria: 
hace más de diez años venimos desarrollando distintas acciones vinculadas a esta materia; hay una concordancia entre la visión y los planes estratégicos (Participante 7, Universidad C).

La Universidad C cuenta con dos direcciones dependientes de la Vicerrectoría Académica. Ambas trabajan articuladamente e inclusive comparten el mismo director. Una de ellas cumple la misión de: "Apoyar los programas de pregrado y posgrado en el uso de las Tecnologías de la Información y Comunicaciones (TIC), con el fin de potenciar las prácticas educativas y los procesos de enseñanza aprendizaje", mientras que la otra tiene la misión de: "Asegurar la calidad de los procesos formativos de pregrado, orientando y colaborando, fundamentalmente en la innovación y el diseño curricular, en los procesos de aseguramiento de calidad de carreras y programas, así como en la calidad de la docencia y evaluación para la docencia universitaria". La convergencia entre ambas direcciones ha reforzado la visión institucional de acompañar a los docentes universitarios en el desarrollo de dispositivos de formación híbridos, tomando en cuenta tanto los aspectos técnicos, como los materiales, humanos y pedagógicos implícitos en el proceso.

\section{b. Cultura tecnológica}

Esta categoría fue entendida según la definición de Hy-Sup (Deschryver \& Charlier, 2012) como los elementos del discurso expresados en términos de moda, cultura, presión colectiva, asociados al concepto de b-learning o al desarrollo de dispositivos de formación híbridos.

Para la unanimidad de los entrevistados el estar inmersos en una cultura tecnológica, es una presión externa que incita a las universidades a actualizar sus formaciones, integrando las tecnologías digitales.

Uno ve que los estudiantes dicen por qué en tal universidad las asignaturas son virtuales y nosotros no las tenemos todavía, entonces ellos piden que uno avance en la tecnología (Participante 1, Universidad A). 
Los estudiantes empiezan 'profe, por qué usted no sube los materiales como otro profesor' (Participante 2, Universidad B).

Existe una demanda creciente con respecto al uso de las tecnologías en la Universidad (...) modernizar la práctica educativa hoy día es usar tecnologías (Participante 8, Universidad C).

\section{c. Gestión de la calidad y evaluación de la enseñanza- aprendizaje}

La calidad de este tipo de formaciones es un tema que emerge de manera recurrente en el discurso de los entrevistados, asociada a objetivos diferentes de acuerdo con la institución de pertenencia. En dos universidades la calidad estaría supeditada a criterios y/o presiones externas, sin que exista un proceso de reflexión institucional acerca de cuál es el valor agregado de los dispositivos de formación híbridos en la educación superior. Así, por ejemplo, para los entrevistados de la Universidad A, estos deben tener directa relación con los estándares de calidad que defina el Consejo Nacional de Acreditación (CNA) y que aún no han sido claramente explicitados.

Está la acreditación institucional y tiene ciertos ejes de evaluación (...) dentro de estos ejes determinaron una columna que se llama criterios específicos para la formación virtual (...) La CNA ha sacado a consulta esos criterios y yo sé que debería sacar estándares este año (...) si uno va a avanzar en la modalidad necesita suscribirse a unos estándares y nosotros hemos mirado estándares que son internacionales (Participante 1, Universidad A).

¿Cuáles son los criterios que está poniendo la CNA, respecto de la docencia de pregrado y el uso de tecnologías? ¿Cuáles son los criterios de acreditación? (Participante 5, Universidad A).

Por su parte, en la Universidad B los entrevistados consideran que el interés reciente de las autoridades por promover el desarrollo de asignaturas en modalidad b y e-learning en pregrado se debe a "la competencia y las nuevas exigencias externas" (Participante 6, Universidad B). 
En la Universidad C, la calidad de los dispositivos de formación híbridos se evalúa con criterios específicos, relativos al ejercicio docente. Además, se desarrollan estudios con el objetivo de evaluar y dar seguimiento a los resultados de la puesta en marcha de estas formaciones. El fin último de la calidad para esta universidad es la mejora de los aprendizajes de los estudiantes. Por lo que los análisis que realizan de los resultados les permiten tomar decisiones para implementar nuevas acciones institucionales:

tenemos que dar cuenta si todo este set de apoyo ayuda o favorece los procesos de enseñanza y aprendizaje, entonces no basta con hacer la implementación, sino que creemos que esto es un círculo, que tiene que ver con distintas etapas y fases (Participante 8, Universidad C).

En la Universidad A, el desarrollo de investigaciones en torno a los aprendizajes de los estudiantes universitarios como resultado de su participación en formaciones híbridas es percibido como una necesidad:

Necesitamos saber las diferencias entre un docente que fue acompañado en el aula con respecto a uno que no lo fue (...) Me gustaría saber si hay diferencia entre un profesor que ha incorporado tecnologías y otro que no, en relación con la enseñanza-aprendizaje, y por ende que demuestre si hay mejor rendimiento (Participante 5, Universidad A).

Además, la mayoría de los entrevistados de las universidades A y B se mostraron interesados en comenzar a obtener informes que les puedan dar luces acerca de la calidad de los dispositivos de formación híbridos y no tan solo de su uso, que es lo que producen y/o reciben actualmente.

Los reportes no son específicos (...) son reportes de consumo, respecto a ingresos a la plataforma, de estudiantes, docentes, cantidad de documentos (...) contamos cuánto se descarga y las visitas a las herramientas de comunicación como el foro (Participante 1, Universidad A). 
nos piden reportes para saber cuántos profesores de su departamento están utilizando plataformas, pero estamos todavía en el tema del uso (...) ¿cuántos son? (Participante 4, Universidad B).

lo que hoy necesitamos es información consolidada respecto de las tendencias en nuestra aula virtual, de manera de ir colocando focos de desarrollo (Participante 3, Universidad A).

En cuanto a la evaluación de los aprendizajes de los estudiantes, los entrevistados perciben efectos positivos que podrían potenciarse a través del uso de entornos virtuales de aprendizaje. La ventaja más mencionada es la facilidad para otorgar una rápida retroalimentación y acompañamiento de los estudiantes:

[en el aula virtual] es mejor la retroalimentación, es más rápido el tema de la evaluación, eso le facilita el trabajo al profe... Además, incluye rúbricas, tú puedes crear tu rúbrica y entonces el alumno sabe en qué se equivocó cuando no le va bien y te muestra dónde el profe evaluó (Participante 4, Universidad B).

Se pueden hacer ayudantías sincrónicas, puedes disponer las guías, hacer que los estudiantes practiquen. Tener ahí mismo los resultados, tomar acciones remediales con quienes tengan un nivel más bajo (...) hacer todo eso en presencial es muy lejano para nosotros (Participante 1, Universidad A).

Por otro lado, consideran que la utilización de entornos virtuales de aprendizaje facilitaría el desarrollo y evaluación de competencias digitales de los estudiantes de pregrado:

Estamos avanzando en el desarrollo de hitos evaluativos, en los que vamos a medir los niveles de logro respecto de las competencias tecnológicas (Participante 3, Universidad A).

En la Universidad C, la evaluación de los aprendizajes se realiza a través de las propias percepciones de los estudiantes acerca de la experiencia y eso les sirve además para relevar buenas prácticas docentes: 
Muchas de las buenas prácticas las obtenemos de entrevistas a estudiantes, les preguntamos si él pudo tener mejores resultados de aprendizaje dado que su profesor usó de mejor manera el aula virtual (Participante 7, Universidad C).

Sin embargo, recién este año esta información obtenida a través de cuestionarios y entrevistas a los estudiantes podrá ser sistematizada, gracias a una investigación en curso:

Queremos saber cómo está incidiendo esto en su proceso de aprendizaje y cómo esto se relaciona con la forma en que los profesores lo usan [el aula virtual] (...) estamos en la primera fase [de la investigación] (Participante 9, Universidad C).

\section{d. Apoyo financiero}

En las tres instituciones el desarrollo de dispositivos de formación híbridos está supeditado a los recursos financieros, materiales y humanos que la Vicerrectoría Académica dispone o no para ello. En dos de las tres instituciones la estrategia se fue dando de "abajo hacia arriba", es decir, primero surgieron iniciativas individuales (de ciertas Facultades, decanos y/o académicos), y más precisamente, a través de proyectos de innovación internos y/o externos. Luego las autoridades comenzaron a apoyarlos y a interesarse por comenzar la discusión en torno a las acciones y estrategias a implementar en toda la institución:

hay una estrategia que va como desde abajo hacia arriba que tiene relación con las iniciativas propias de las facultades (Participante 1, Universidad A).

en los últimos años las facultades por sus propios proyectos de desarrollo han venido incorporando el tema tecnológico y de la formación híbrida (Participante 3, Universidad A).

este año la unidad se adjudicó un proyecto de la Vicerrectoría Académica para potenciar la virtualización de asignaturas (Participante 4, Universidad B). 
En la Universidad C, a diferencia de las otras dos instituciones, el apoyo financiero de la Vicerrectoría Académica al desarrollo de estos dispositivos de formación es parte de la planificación estratégica de la institución y se ha ido incrementando con los años:

No solo hemos crecido en apoyo de permanencia de las personas, sino en la formación de equipos, que son recursos (...) ha sido un apoyo constante en base a los resultados (Participante 7 , Universidad C).

\section{e. Apoyo técnico-pedagógico}

El apoyo financiero repercute en las acciones implementadas en las tres instituciones, las que en los últimos años se han focalizado principalmente en la generalización del uso de las plataformas virtuales de aprendizaje y en la capacitación y/o acompañamiento a los docentes y estudiantes para su uso. Solo una de las tres universidades focaliza el acompañamiento de los docentes en mejorar las competencias técnicas y pedagógicas del uso de la o las plataformas virtuales de aprendizaje. No obstante, la preocupación por el acompañamiento pedagógico es el desafío al que quieren avanzar las otras dos instituciones.

Las capacitaciones son más bien instrumentales (Participante 2, Universidad B).

nuestros cursos se han orientado más a la parte técnica, pero creo que ahora es necesario potenciar lo pedagógico (Participante 4: Universidad B).

desde este año todos los docentes nuevos tienen que tomar un curso básico, donde les contamos cuáles son las plataformas, cómo se accede, dónde están los recursos de aprendizaje, la importancia de la comunicación online, etc. (Participante 1, Universidad A).

Así mismo, en la Universidad A existen "coordinadores", cuya misión es acompañar a los docentes y estudiantes en el uso de las plataformas virtuales de aprendizaje. 
Si tienes problemas para acceder al aula virtual, está él ahí disponible para darle la orientación al respecto (Participante 1 , Universidad A).

La Universidad B solo cuenta con un pequeño equipo de profesionales que apoya esta gestión. Al ser entrevistados, interpretan esta sobrecarga como prueba de la desvalorización y la falta de conocimiento por parte de las autoridades de los recursos que se requieren para realizar esta labor.

No están conscientes del trabajo que ello implica y del costo operacional (...) creen que es llegar y así se hace (Participante 2, Universidad B).

la secretaria tiene solo un par de horitas, pero para lo puntual, habilitar una cuenta, crear un curso. No hay asistencia técnicopedagógica del uso de las herramientas (Participante 6, Universidad B).

En tanto, en la Universidad C, se cuenta con una gran diversidad de medios para acompañar a los docentes en el proceso:

nosotros proveemos de plataforma, capacitación, generación de recursos a los docentes, asesorías personalizadas, a través de estudiantes y profesionales, para que respondan a las inquietudes, sobre todo a principio de semestre (...) los mecanismos son todos esos; formación, apoyo, asesoría, apoyo telefónico, vía mail, etc. (Participante 7, Universidad C).

Para los responsables institucionales de esta universidad, una de las acciones de acompañamiento más exitosas es la formación continua de los docentes. Para ello, diversifican los tipos de capacitaciones según el nivel de experiencia en la integración de tecnologías digitales para la enseñanza-aprendizaje de los académicos:

hacemos un proceso formativo permanente en distintas actuaciones, porque hay profesores que son iniciales y hay que darles capacitación inicial, hay profesores ya avanzados (...) que 
muestran evidencia que su práctica está más enriquecida y ahí hacemos el proceso de incentivar la innovación y eso llama a la buena práctica, la que se registra, se valida y se difunde en las redes sociales, para que mucha gente la pueda ver (Participante 7, Universidad C).

Una de las preocupaciones y desafíos que le queda por resolver a esta universidad es la de incentivar a los docentes a honorarios, que representan un alto porcentaje de los académicos de esta institución, a participar en estas capacitaciones:

tenemos una deuda principalmente con los profesores agregados, nuestra universidad cuenta con muchos profesores que no son los profesores jerarquizados y siempre ha sido una deuda incorporarlos más, porque a pesar de que siempre hacemos la difusión, a este grupo de profesores, que es más del 50\%, no tenemos una forma de comprometerlos (Participante 8, Universidad C).

\section{Reconocimiento institucional}

En las tres instituciones la valorización de los docentes que desarrollan dispositivos de formación híbridos es solamente uno de los criterios de reconocimiento a su labor o para ascender en la jerarquía académica. En la Universidad B y C se otorgan premios a la excelencia docente:

Se hace el premio a la excelencia docente, pero eso involucra muchas otras cosas, donde las tecnologías son un punto más dentro del check list (Participante 2, Universidad B).

los profesores pueden, gracias a la evaluación de la docencia, cambiar de jerarquía y en su carrera académica, además la buena docencia en nuestra universidad se premia anualmente con un dispendio económico a los tres mejores profesores de la unidad académica (...) esta evaluación contempla un acápite de uso del aula virtual (Participante 1, Universidad C). 
En tanto en la Universidad C, también se considera la confección de material o la innovación académica, al momento de evaluar la jerarquía de un académico:

En lo general [el desarrollo de dispositivos de formación híbridos] suma, pero no es un elemento que te diga que porque hago esto y me dedico solo a esto, voy a ascender de categoría académica (...) ahora sí, dentro de la calificación, es decir, del desempeño de los académicos, también está considerada la confección de material o la innovación académica (Participante 3, Universidad A).

f. Impacto de los dispositivos híbridos en la movilidad e intercambio interinstitucional

Resulta interesante destacar la ausencia en el discurso de los responsables institucionales chilenos de las categorías de análisis de contenido identificadas en el proyecto Hy-Sup: "Movilidad institucional" y "Colaboración interinstitucional" como posibles efectos positivos del desarrollo de dispositivos de formación híbridos. Esto puede deberse a que la política institucional nacional no promueve masivamente la colaboración entre instituciones y la movilidad internacional como en los países europeos. Para los entrevistados, la colaboración interinstitucional fue mencionada desde un punto de vista más bien utilitario, en el sentido de prestar soporte a través de la plataforma a otras instituciones educativas, como colegios o para mostrarse como referentes en el tema ante otras instituciones y/o buscar ejemplos internacionales de buenas prácticas:

[el entorno virtual de aprendizaje desarrollado por nuestra universidad] es tan abierto, que lo ocupan dos colegios de la ciudad y los profesores cuando hacen asesorías técnicas (Participante 4, Universidad B).

Hemos tenido reconocimiento nacional. Muchas instituciones vienen a conocernos y a saber qué ha pasado con nuestro proceso (Participante 7, Universidad C).

la Vicerrectoría Académica hace muchos viajes y muchas observaciones a otras instituciones a observar cómo es la realidad, 
cuáles son las nuevas medidas que están implementando (...) entonces eso les permite tomar decisiones, aterrizarlas al contexto de esta universidad y ver si son fiables o no de implementar (Participante 9, Universidad C).

\section{g. Usos observados del aula virtual}

En las Universidades A y B los usos observados del aula virtual por los responsables institucionales son principalmente aquellos que cumplen con una función informativa de los contenidos del curso. Se adjuntan principalmente el programa de la clase, presentaciones y documentos textuales:

en estos momentos se está usando mucho como repositorio, es decir, subo las presentaciones, subo el PDF, el Power Point (Participante 6, Universidad B).

lo que hacen es fundamentalmente subir recursos educativos, guías, tutoriales, etc. Ese es el mayor uso que le dan y marginalmente están ocupando la herramienta de quizz o test (Participante 1, Universidad A).

Para los responsables institucionales de la Universidad C, utilizar el aula virtual solo como un repositorio de recursos textuales es identificado como una "mala práctica":

Una mala práctica es que los profesores activan el aula virtual y lo único que hacen es publicar el PPT o avisos para los estudiantes, entonces este espacio se subutiliza (Participante 8, Universidad C).

Cabe destacar que los esfuerzos de esta universidad se focalizan en incentivar la interacción entre los estudiantes y el docente, si bien observan una tendencia a avanzar en ese tipo de usos del aula virtual:

el uso que uno observa y que venimos incentivando son las prácticas formativas con mayor interacción, porque al principio la mayoría de los usos que la gente le da al aula virtual era principalmente como repositorio de materiales (...). Hoy día la tendencia que observamos es aumentar los grados de interacción 
profesor-estudiantes, entendiendo que estamos en una docencia presencial y esto es apoyo a la docencia presencial de pregrado, es un gran valor que esto se produzca (Participante 7, Universidad C).

Uno de los responsables institucionales de la Universidad C valora significativamente el desarrollo de dispositivos de formación híbridos que cuentan con una planificada articulación de la presencia y la distancia, destacando aquello como una buena práctica en pregrado:

una buena práctica es cuando el profesor logra enhebrar de manera muy fina la docencia presencial, con la docencia virtual y no lo ve como un paralelo. Hemos visto cursos donde el profesor con sus materiales logra decir: 'esta parte del curso la vamos a hacer en un aula virtual' y en el aula virtual dice 'este es material para conversar en la clase presencial' y entonces se genera una trenza de diálogo bien interesante entre la presencia y la distancia (Participante 7, Universidad C).

\section{Conclusiones}

En el análisis de los resultados se observa que el desarrollo de los dispositivos de formación híbridos en pregrado es un proceso de largo aliento. Solo en la Universidad C los dispositivos de formación híbridos constituyen una práctica arraigada. En esta universidad se comprendió tempranamente (hace 20 años) que el desarrollo de estas prácticas debía ser fruto de una estrategia institucional basada en el compromiso de todos los actores. En las otras dos universidades, las prácticas analizadas se pueden situar en tete de point, ya que se fomenta el desarrollo institucional de dispositivos de formación híbridos, pero aún no constituyen una práctica generalizada, debido fundamentalmente a que no existe una visión institucional clara ni compartida por todos los actores, lo que repercute a su vez en los escasos recursos materiales y humanos que se asignan a esta área. La investigación europea HySup también evidenció que el desarrollo de dispositivos de formación híbridos tenía en sus inicios un débil apoyo institucional: "la voluntad institucional de apoyar el desarrollo de este tipo de dispositivos se observa principalmente en las discusiones, pero no se aprecian acciones 

EN CARRERAS DE PREGRADO DE TRES UNIVERSIDADES CHILENAS - P. Costa, K. Celis, N. Castillo-Valenzuela, G. Espinoza

concretas" (Deschryver \& Charlier, 2012, p. 231).

Todas las universidades reconocen la necesidad de acompañar técnica y pedagógicamente a los docentes para incentivar el desarrollo de dispositivos de formación híbridos. Las diferencias están en los recursos y acciones que cada universidad destina para ello. En las universidades chilenas analizadas, en las que los dispositivos de formación híbridos no constituyen una práctica arraigada, se han concentrado en facilitar la apropiación tecnológica del aula virtual por parte de docentes y estudiantes, y no se observan acciones que acompañen a los docentes pedagógicamente. Como consecuencia de ello, los usos observados del aula virtual son mayoritariamente como repositorio de documentos textuales. La investigación Hy-Sup, caracteriza este tipo de dispositivos de formación como orientados hacia la enseñanza y los denomina como "la escena".

A partir del contraste de los resultados, se observó que contar con una visión y estrategias institucionales claras y definidas impacta positivamente en la asignación de recursos tanto materiales como humanos para apoyar la implementación de los dispositivos de formación. Esto se traduce, a su vez, en un interés por fomentar la reflexión pedagógica y por generar estudios que analicen el impacto del desarrollo de dispositivos de formación híbridos en los aprendizajes de sus estudiantes. Así, por ejemplo, en el contexto europeo, analizado por Douzet et al. (2012) los servicios de acompañamiento — para el desarrollo de dispositivos de formación híbridos en las universidades- están generalmente asociados a un equipo de docentes-investigadores especialistas en pedagogía universitaria. De las tres universidades chilenas analizadas, solo una comenzó a asociar recientemente el acompañamiento tecnopedagógico de los docentes con el desarrollo de una investigación que da seguimiento a estas actividades.

En general se aprecia una falta de reflexión de parte de los responsables institucionales acerca de los aportes pedagógicos de este tipo de formaciones. Las razones evocadas para integrarlas provienen de demandas externas (acreditación) o como parte de una cultura 
tecnológica en la que está inmersa la sociedad, sin que se defina cuál es su valor intrínseco. A diferencia del contexto europeo, donde los responsables institucionales consideran que la definición de una visión institucional debe ser "el fruto de un proceso de reflexión de las universidades y acompañado por una política concertada y práctica a nivel interuniversitario, regional, nacional e incluso europeo" (Douzet et al., 2012, p. 230).

De esta manera, el aporte en términos de hipótesis de la investigación es la relación atisbada entre la planificación estratégica institucional y los efectos positivos en el desarrollo de dispositivos de formación híbridos, entendidos como un proceso de causalidad circular. Esto, abre perspectivas teóricas que guíen el análisis de los efectos de este tipo de formaciones en los aprendizajes de los estudiantes.

Si bien no es posible generalizar los resultados de esta investigación, se recomienda fomentar a mayor escala el desarrollo de estudios que permitan generar procesos de reflexión interna en las instituciones de educación superior, así como monitorear los cambios desde una perspectiva sistémica, teniendo en cuenta tanto aspectos técnicos como pedagógicos, individuales y contextuales. Asimismo, estos análisis se verían enriquecidos a través de la colaboración interinstitucional nacional e internacional y permitirían avanzar en la elaboración de estándares de calidad de estas formaciones, con acciones a corto, mediano y largo plazo, sustentadas por la evidencia empíricas y las necesidades locales.

\section{Referencias}

Agamben, G. (2011). ¿Qué es un dispositivo? Sociológica, 26(73), 249-264. Recuperado de http://www.scielo.org.mx/pdf/soc/v26n73/v26n73a10. pdf

Albero, B. (2010). Penser le rapport entre formation et objets techniques. Repères conceptuels et épistémologiques. En G. Leclercq \& R. Varga (Eds.), Dispositifs de formation et environnements numériques, enjeux pédagogiques et contraintes informatiques (pp. 1-22). Paris: Lavoisier. Recuperado de https://edutice.archives-ouvertes.fr/edutice-00578355/ document 

EN CARRERAS DE PREGRADO DE TRES UNIVERSIDADES CHILENAS - P. Costa, K. Celis, N. Castillo-Valenzuela, G. Espinoza

Albero, B. (2014). La pédagogie à l'université entre numérisation et massification. En Lameul,G; Loisy, C (Ed.), La Pédagogie universitaire à l'heure du numérique (pp. 25-53). Bruxelles: De Boeck.

Albero, B., Linard, M., \& Robin, J. (2009). Petite fabrique de l'innovation à l'université. Quatre parcours de pionniers. Paris: Harmattan.

Area, M., San Nicolás, M., y Sanabria, M. (2018). Las aulas virtuales en la docencia de universidad presencial: la visión del alumnado. RIED Revista Iberoamericana de Educación a Distancia, 21(2). Recuperado de http://revistas.uned.es/index.php/ried/article/view/20666

Arfuch, L. (2002). El espacio biográfico: dilemas de la subjetividad contemporánea. Buenos Aires: Fondo de Cultura Económica.

Bartolomé, A. (2008). Entornos de aprendizaje mixto en educación superior. Revista Iberoamericana de Educación a Distancia, 1(11), 15-51.

Berg, B. L. (2009). Qualitative research methods for the social sciences (7th ed.). Boston: Allyn \& Bacon.

Bonamy, J., Charlier, B., \& Saunders, M. (2002). Issues in the organizational and change context for case study courses. Namur: Facultés Universitaires Notre-Dame de la Paix.

Burton, R., Borruat, S., Charlier, B., Coltice, N., Deschryver, N., Docq, F, ... Villiot-Leclerq, E. (2011). Vers une typologie des dispositifs hybrides de formation en enseignement supérieur. Distance et Savoir, 9(1), 6996. https://doi.org/10.3166/ds.9.69-96

Charlier, B. (2019). Les environnements numériques d'apprentissage: Quelques éléments d'intelligibilité pour la e-Formation. En A. Jézégou (Ed.), Traité de la e-Formation des adultes (pp. 89-117). Louvain-laNeuve, Belgique : De Boeck.

Charlier, B., Deschryver, N., \& Peraya, D. (2006). Une définition des dispositifs hybrides. Distance et Savoir, 4(4), 469-496.

Cher, L. \& Wang, L. (2016). Blended learning for quality higher education. Selected cas studies on implementation from Asia-Pacific. París: Unesco.

Costa, P., Peraya, D., y Rizza, C. (octubre, 2016). Análisis de los usos del libro de clases digital en el contexto escolar francés. Trabajo presentado en el VI Encuentro: conferencias chilenas en tecnologías del aprendizaje, Chillán, Chile. Recuperado de http://archive-ouverte.unige.ch

Coyne, I. (1997). Sampling in qualitative research. Purposeful and theoretical sampling; merging or clear boundaries? Journal of Advanced Nursing, 26(3), 623-630. https://doi.org/10.1046/j.1365-2648.1997.t01-2500999.x 
Davis, F. D. (1985). A technology acceptance model for empirically testing new end-user information systems: Theory and results (Tesis doctoral). Massachusetts Institute of Technology, Cambridge, MA. Recuperado de http://dspace.mit.edu/handle/1721.1/15192

Delgado-Almonte, M., Andreu, H., \& Pedraja-Rejas, L. (2010). Information technologies in higher education: Lessons learned in industrial ingenieering. Journal of Educational Technology and Society, 13(4), 140154.

Deschryver, N. (2008). Interaction sociale et expérience d'apprentissage en formation hybride (Tesis doctoral). FAPSE, section Sciences de l'Éducation, Université de Genève, Genève. Recuperado de http://tecfa. unige.ch/perso/deschryv/doc/these_deschryver.pdf

Deschryver, N. \& Charlier, B. (2012). Dispositifs hybrides, nouvelle perspective pour une pédagogie renouvelée de l'enseignement supérieur. Recuperado de http://prac-hysup.univ-lyon1.fr/spiral-files/download?mode=inlin e\&data $=1757974$

Douzet, C., Letor, C., \& Ronchi, A. (2012). Dispositifs hybrides et institution. Recuperado de http://prachysup.univlyon1.fr/webapp/website/website. html?id=1578544\&pageId=1751

Garrrido, R. (2009). B-learning como solución al problema de recursos académicos escasos en educación superior (Tesis de Magíster). Facultad de Ciencias Físicas y Matemáticas, Universidad de Chile, Santiago de Chile.

Glazer, F. (2011). New pedagogies and practices for teaching in higher education: Blended learning: Across the disciplines, across the academy. Sterling: Stylus.

Jovanovic, J., Gasevic, D., Dawson, C., \& Mirriahi, N. (2017). Learning analytics to unveil learning strategies in a flipped classroom. The Internet and Higher Education, 33, 74-85. https://doi.org/10.1016/j. iheduc.2017.02.001

Karsenti, T., Larose, F., y Núñez, M. (2002). La apertura universitaria a los espacios de formación virtual: un reto a la autonomía estudiantil. Revista Electrónica de Investigación Educativa, 4(1). Recuperado de https://www.researchgate.net/publication/28054583_La_apertura_ universitaria_a_los_espacios_de_formacion_virtual_Un_reto_a_la_ autonomia_estudiantil

Lai, P. C. (2017). The literature review of technology adoption models and theories for the novelty technology. Journal of Information Systems and Technology Management, 14(1), 21-38. https://doi.org/10.4301/S180717752017000100002 
250 ANÁLISIS DE LA IMPLEMENTACIÓN INSTITUCIONAL DE LA MODALIDAD B-LEARNING EN CARRERAS DE PREGRADO DE TRES UNIVERSIDADES CHILENAS - P. Costa, K. Celis, N. Castillo-Valenzuela, G. Espinoza

Lamine, H. \& Abdid, H. (abril, 2016). Vers une nouvelle tendance de l'innovation pédagogique basée sur la classe inversée. Trabajo presentado en RAPU, Tunisie, Francia.

Latour, B. (1988). Science in action. Recuperado de http://www.hup.harvard. edu/catalog.php?isbn=9780674792913

Lebrun, M., Gilson, C., \& Goffinet, C. (2017). Vers une typologie des classes inversées. Education et Formation, 306, 125-146.

Léontiev, A. (1975). Activity, conscience and personality. Englewood Cliffs, NJ: Prentice-Hall.

Long, T., Cummins, J., \& Waugh, M. (2016). Use of the flipped classroom instructional model in higher education: instructors' perspectives. Journal of Computing in Higher Education, 29(2), 179-200. https://doi. org/10.1007/s12528-016-9119-8

Lucke, T. (2014). Using learning analytics to evaluate the effectiveness of the flipped classroom approach. Trabajo presentado en Australasian Association for Engineering Education (AAEE). Wellington NZ. Recuperado de https://www.researchgate.net/publication/270162113_ Using_Learning_Analytics_to_Evaluate_the_Effectiveness_of_the_ Flipped_Classroom_Approach

Marín, V., Duart, J., Galvis, A., \& Zawacki-Richter, O. (2018). Thematic analysis of the international journal of educational Technology in Higher Education (ETHE) between 2004 and 2017. International Journal of Educational Technology in Higher Education, 15(1). https:// doi.org/10.1186/s41239-018-0089-y

Martín García, A., García del Dujo, A., y Muñoz, J. (2014). Factores determinantes de adopción de blended learning en educación superior. Adaptación del modelo UTAUT. Educación XX1, 17(2), 217-240. https://doi.org/10.5944/educxx1.17.2.11489

Mazohl, P. \& Makl, H. (2016). Quality criteria for the institution. En Mazohl,P, Makl,H, Breitenecker, F, Koerner, A, Winkler, S. (Eds.), Blended learning quality-concept optimized for adult education (pp. 3750). Austria: Lifelong Learning Programme, Blended Learning Quality.

Means, B., Toyama, Y., Murphy, R., \& Baki, M. (2013). The effectiveness of online and blended learning: A meta-analysis of the empirical literature. Teachers College Record, 115(3), 1-47.

Mejía, C., Michalón, D., Michalón, R., López, R., Palmero, D., y Sánchez, S. (2017). Espacios de aprendizaje híbridos. Hacia una educación del futuro en la Universidad de Guayaquil. Medisur, 15. Recuperado de http://scielo.sld.cu/scielo.php?script=sci_arttext\&pid=S1727897X2017000300010 
Mendiburo-Seguel, A. y Reininger, M. (2011). Investigación comparativa sobre las evaluaciones de estudiantes universitarios frente a dos modelos de E-Learning. Calidad en la Educación, 34, 103-122. https:// doi.org/10.4067/s0718-45652011000100005

Navarrete, L., Aburto, G., y Fuentes, R. (octubre, 2016). Modelo TIC para el Sistema de Inducción y Adaptación a la Vida Universitaria: caso de la Universidad del Bío-Bío. Trabajo presentado en VI Encuentro: Conferencias chilenas en tecnología y aprendizaje. Chillán, Chile. Recuperado de http://www.ubiobio.cl/tecnologia/

Nguyet, A., Zhu, Y., Struyven, K., \& Blieck, Y. (2016). Who or what contributes to student satisfaction in different blended learning modalities? British Journal of Educational Technology, 48(2), 473-489. https://doi.org/10.1111/bjet.12431

Peraya, D. (2015). Professionnalisation et développement professionnel des enseignants universitaires: une question d'actualité. Distances et Médiations des Savoirs, 10. Recuperado de https://dms.revues.org/1094

Peraya, D. \& Campion, B. (2007). L'analyse des dispositifs hybrides: les effets d'un changement d'environnement virtuel de travail: d'un site Web à la plateforme Claroline. En M. Frenay, P. Wouters, \& B. Raucent (Eds.), Les pédagogies actives: enjeux et conditions. Actes du 4eme colloque Questions de pédagogies dans l'enseignement supérieur (pp. 447-456). Louvain-La-Neuve: Presses universitaires de Louvain.

Peraya, D., Charlier, B., \& Deschryver. N. (2006). Lanalyse des dispositifs techno pédagogiques: comment rendre compte des effets de l'introduction des innovations? Actes du colloque CEMAFORAD 3. Trabajo presentado en Colloque Euro-Méditerranéen et Africain sur la Formation à Distance: Intégration des TIC à l'enseignement et à la formation. Réalités et Perspectives, Sousse Túnez.

Peraya, D., Charlier, B. \& Deschryver, N. (2014). Une première approche de l'hybridation. Étudier les dispositifs hybrides de formation. Pourquoi? Comment? Éducation et formation, 301, 15-34.

Peraya, D. \& Peltier, C. (mayo, 2012). Une année d’immersion dans un dispositif de formation aux technologies. Prise de conscience du potentiel éducatif des TICE, intentions d'action et changement de pratique. Trabajo presentado en Colloque scientifique international sur les TIC en éducation, Montréal, Canadá.

Pierrot, L., El-Kechai, H., Iksal, S. \& Cerisier, J. (2017). Étude de la circulation des pratiques numériques juvéniles: approche par la prescription de l'observation. Actes de la Conférence EIAH 2017, 77-88. Recuperado de https://www. researchgate.net/profile/Bruno_De_Lievre/publication/321240409_ 
252 ANÁLISIS DE LA IMPLEMENTACIÓN INSTITUCIONAL DE LA MODALIDAD B-LEARNING EN CARRERAS DE PREGRADO DE TRES UNIVERSIDADES CHILENAS - P. Costa, K. Celis, N. Castillo-Valenzuela, G. Espinoza

Actes_de_la_Conference_EIAH_2017_8eme_Conference_sur_les_ Environnements_Informatiques_pour_1\%27Apprentissage_Humain/ links/5a7312e80f7e9b20d48ee8b0/Actes-de-la-Conference-EIAH2017-8eme-Conference-sur-les-Environnements-Informatiques-pourlApprentissage-Humain.pdf

Porter, W., Graham, C., Bodily, R., \& Sandberg, D. (2016). A qualitative analysis of institutional drivers and barriers to blended learning adoption in higher education. Internet and Higher Education, 28, 17-27.

Quintin, J. (2008). Accompagnement tutorial d'une formation collective via Internet. Analyse des effets de cinq modalités d'intervention tutorial sur l'apprentissage en groupes restreint (Thèse en vue de l'obtention d'un doctorat en Sciences de l'Éducation, Université de Mons- Hainaut et Université Stendhal-Grenoble 3). Recuperado de http://tel.archivesouvertes.fr/docs/00/34/90/13/PDF/Quintin_2008_These2.pdf

Rabardel, P. (1995). Les hommes et les technologies. Approche cognitive des instruments contemporains. Paris: Armand Collin.

Rivera-Laylle, L., Fernández, K., Guzmán, F., y Pulido, J. (2017). La aceptación de las TIC por profesorado universitario: conocimiento, actitud y practicidad. Revista Electrónica Educare del Centro de Investigación y Docencia en Educación de la Universidad Nacional Costa Rica, 21(3), 1-18. https://doi.org/10.15359/ree.21-3.6

Sanabria, A., Castro, F., Padrón, J., y Area, M. (2013). La opinión del profesorado y del alumnado sobre el uso de las aulas virtuales en la metodología b-learning. Revista Fuentes, 13, 117-138.

Sayago, S. (2014). El análisis del discurso como técnica de investigación cualitativa y cuantitativa en las ciencias sociales. Cinta de Moebio, 49, 1-10. https://doi.org/10.4067/S0717-554X2014000100001

Schwarzenberg, P., Navon, J., Nussbaum, M., Pérez-Sanagustín, M., \& Caballero, D. (2017). Learning experience assessment of flipped courses. Journal of Computing in Higher Education, 30(2), 237-258. https://doi.org/10.21125/iceri.2017.1521

Swan, J. \& Scarbrough, H. (2001). Knowledge management: Concepts and controversies. Journal of Management Studies, 38(7), 913-921. https:// doi.org/10.1111/1467-6486.00265

Tsoukas, H. \& Vladimirou, E. (2001). What is organizational knowledge? Journal of Management Studies, 38(7), 973-993. https://doi. org/10.1111/1467-6486.00268

Turpo, O. (2012). La modalidad educativa blended learning en las universidades de Iberoamérica: análisis y perspectivas de desarrollo. Educar, 48(1), 123-147. 
Van der Linden, K. (2014). Blended learning as transformational institutional learning. En Ch. Hanson (Ed.), 165. New directions for higher education (pp. 75-85). Recuperado de: https://onlinelibrary.wiley.com/ doi/10.1002/he.20085

Vásquez, M. (2014). Modelos blended learning en educación superior: análisis crítico-pedagógico (Tesis doctoral). Universidad de Salamanca, Salamanca, España. Recuperado de http://gredos.usal.es/jspui/ bitstream/10366/127936/1/DTHE_VasquezAstudilloM_BlendedLear ningEducacionSuperior.pdf

Vásquez-González, B., Pleguezuelos, C., y Cabas, J. (mayo, 2011). B-learning en la Universidad del Bío Bío, Chile: la experiencia del programa de magister en agronegocios. Trabajo presentado en II Seminario de Innovación Docente en la Enseñanza Superior del Desarrollo Rural en Iberoamérica, Almería, España.

Vygotski, L. (1985). Pensée et Langage (Réedition). Paris: La Dispute.

Yi, L. (2018). Current problems with the prerequisites for flipped classroom teaching---a case study in a university in Northwest China. Smart Learning Environments, 5(1), 1-23. https://doi.org/10.1186/s40561018-0051-4 